\title{
¿Cómo perciben los hijos la crianza materna y paterna? Diferencias por edad y sexo ${ }^{(1)}$
}

\author{
Miguel Ángel Rodríguez, María Victoria Del Barrio, Miguel Ángel Carrasco \\ Facultad de Psicología \\ Universidad Nacional de Educación a Distancia
}

Disponible online 30 de abril de 2009

\begin{abstract}
Con el objetivo de analizar la percepción sobre la crianza materna y paterna, 1182 niños y adolescentes (45,3\% chicos y 54,7\% chicas) con edades comprendidas entre 8 y 16 años, fueron evaluados mediante el Child Report Parental Behaviour Inventory (CRPBI; Shaefer, 1965). Los resultados muestran una mayor sensibilidad de las chicas a la afectividad y en los chicos al control y la hostilidad parentales. La madre obtiene mayores puntuaciones en todos los factores con la excepción de hostilidad. A medida que los hijos crecen, éstos informan de un decremento en el afecto, la comunicación y la supervisión, en ambos padres, y por el contrario, de un incremento en la percepción de la hostilidad.

Palabras clave:

Crianza, niños, adolescentes, conducta parental.

This study focuses on perceived parenting characteristics by children and adolescent. The Child Report Parental Behaviour Inventory (CRPBI; Schaefer, 1965) was applied to a sample of 1182 subjects ( $45.3 \%$ boys and $54.7 \%$ girls) between 8-16 years old. According to children' report, girls are more sensible to parents' affectivity and communication whereas boys are to parental control and hostility. Mothers scored more than fathers in all domains except in hostility. Perception of affectivity, communication and supervision decreases with informants' age and, in contrast, hostility increases.
\end{abstract}

Keywords:

Rearing, children, adolescent, affectivity, hostility, parental behaviour.

\footnotetext{
(1) Esta investigación ha sido subvencionada por el Ministerio de Ciencia y Tecnología dentro de la convocatoria I+D+I, 2001-2004 mediante el proyecto BSO2001 3042I.

Correspondencia: Miguel Ángel Carrasco. Dpto. Personalidad, Evaluación y Tratamiento Psicológico. Facultad de Psicología. UNED. C/ Juan del Rosal, 10. Ciudad Universitaria. 28040 Madrid (Spain). Teléfono: (+34) 9139882 31. Fax: (+34) 9139862 98. E-mail: macarrasco@psi.uned.es
} 
Numerosos autores subrayan la importancia que la percepción de los hijos tiene sobre los hábitos de crianza de sus padres (Grusec y Goodnow, 1994; Ivanova e Israel, 2006; Roa y Del Barrio, 2002). La información obtenida por los hijos, se ha mostrado no sólo fiable (Carrasco, Holgado y del Barrio, 2007; Samper, Cortés, Mestre, Nácher y Tur, 2006), sino también más coherente que la suministrada por sus padres en relación con otras fuentes (González, Cauce y Mason, 1996; Morris, et al, 2002) y con diversos constructos de ajuste emocional en el niño (e.g., rendimiento escolar, sintomatología depresiva, problemas exteriorizados) (Paulson, 1994; Roa y Del Barrio, 2002; Schaefer, 1965). Más aún, Gaylord, Kitzmann y Coleman (2003) encontraron que la información suministrada por los hijos sobre la crianza de los padres posee una mayor validez predictiva sobre el grado de aceptación entre sus iguales, frente a la información aportada por sus padres. La razón que puede explicar esta diferencia es que la información suministrada por los padres parece estar sometida a una mayor deseabilidad social (Roa y Del Barrio, 2001, 2002).

En cualquier caso, en la mayoría de los trabajos realizados con población infantil se muestra que la concordancia entre las distintas fuentes de información es de moderada a baja (Achenbach y McDonough, 1987; Cole, Martin, Powers y Truglio, 1996; Del Barrio, 1990, 2002; Epkins, 1993; Jané, Araneda, Valero y Doménech, 2000; Klein, 1991; Lanz, Scabini, Vermulst y Gerris, 2001) y, particularmente, en lo relativo a los hábitos de crianza (Gaylord et al., 2003; Paulson y Sputa, 1996; Smetana, 1995). Por tanto, el análisis de la perspectiva infantil sobre la crianza es necesario y está justificado, no sólo para garantizar la información obtenida, sino para consolidar la validez de constructo, al que dicho análisis contribuye, así como para contrastar las diferencias aportadas respecto de otras fuentes.

Una gran parte de la investigación coincide en hallar diferencias en la percepción que los hijos tienen de los estilos de crianza del padre y de la madre (Collin y Russell, 1991; Pipp, Shaver, Jenning, Lamborn y Fischer, 1985; Rodrigo et al., 2005; Shek, 1998). Hijos e hijas, tanto en la niñez como en la adolescencia, perciben a la madre más positivamente que al padre en lo que respecta a los aspectos emocionales y a los aspectos democráticos de la crianza (Klein y O’Bryant, 1996; Paulson y Sputa, 1996; Shek, 2000). Las madres, a diferencia de los padres, son más aceptadas (Forehand y Nousiainen, 1993) y son percibidas por los hijos como más comprensivas (Youniss y Smollar, 1985), más cercanas (Paulson, Hill y Holmbeck, 1991), más cariñosas, más controladoras, más implicadas en las tareas escolares de casa (Paulson y Sputa, 1996), con estilos de disciplina más flexibles e igualitarios (Pipp et al., 1985), menos severas (Sorbring, Rödholm-Funnemark y Palmerus, 2003) y más centradas en el hijo/a (Samper et al., 2006). En cambio, los padres son percibidos como más autoritarios, rígidos, severos, restrictivos y formales (Collins y Russell, 1991; Klein y O'Bryant, 1996; Paulson y Sputa, 1996; Shek, 1995,
1998, 2000). Los hijos informan que el padre utiliza métodos de disciplina más severos (Sorbring et al., 2003) y están claramente orientados a proporcionar consejos prácticos y guías de comportamiento (Youniss y Smollar, 1985).

Sin embargo, cuando se estudian las diferencias de estas percepciones en función del sexo de los hijos, los resultados dejan de ser tan homogéneos. La literatura recoge cierta controversia acerca de cómo el sexo de los hijos condiciona la percepción de los estilos de crianza parentales.

Algunos estudios siguen encontrando claras semejanzas (Forehand y Nousiaien, 1993; Musitu y Cava, 2001; Shek, 1998; Smetana 1995), otros indican sólo pequeñas diferencias (Dornbusch, Ritter, Herbert, Roberts y Fraleigh, 1987); otros, en cambio, hallan claras e importantes diferencias en función del sexo del hijo (Gaylord et al., 2003; Mestre, Samper, Tur y Díez, 2001; Shek, 2000). En aquellos trabajos en los que aparecen diferencias relevantes, los varones perciben a sus padres más estrictos (Gaylord et al., 2003; Sorbring et al., 2003) y negligentes que sus hermanas (Hines, Kantor y Holt, 2006). Las mujeres, por el contrario, perciben a sus madres más afectuosas (Younis y Smollar, 1985), y menos estrictas que sus hermanos. Todo lo cual apoyaría el papel mediador del sexo en la percepción del estilo educativo de los padres (Sorbring et al., 2003). Conclusión que queda sustentada por resultados procedentes de otras culturas como la asiática (Shek, 1998, 2000).

El análisis de los datos se complica cuando se considera la edad. En general, los resultados hallados sostienen que a medida que los hijos crecen perciben una disminución del apoyo e implicación en ambos padres (Litovsky y Dusek, 1985; Paikoff y Brooks-Gunn, 1991; Paulson y Sputa, 1996; Shek, 2000; Spera, 2005; Steinberg, 2001) y se advierte también un menor grado de control y supervisión (Litovsky y Dusek, 1985; Paikoff, Brooks-Gunn, 1991 Musitu y Cava, 2001), cosa, por otra parte, perfectamente coherente con el proceso de desarrollo del niño. Algunos investigadores precisan que, cuando los hijos crecen, los padres utilizan, en mayor medida, estrategias disciplinarias inductivas, basadas en el razonamiento, en la interacción verbal y en el manejo de reforzadores, en detrimento de estrategias disciplinarias autoritarias basadas en la interacción física, en la imposición o el poder (Furman y Buhrmester, 1992; McNally, Eysenberg y Harris, 1991; Musitu y Cava, 2001).

La ausencia de resultados concluyentes sobre esta controversia relativa a la mediación del sexo en la percepción de la crianza materna y paterna, así como el efecto de la edad sobre la misma, justifica la necesidad de realizar nuevos estudios al respecto. Por otra parte, es obvio que la sociedad en los últimos veinticinco años ha sufrido extraordinarios cambios socioeconómicos de diversa índole que afectan a la identificación del rol sexual, y de los que cabría esperar un reflejo en la socialización de los hijos y, especialmente, en los posibles cambios relativos a una educación más igualitaria (Lanz, Scabini, Vermulst y Gerris., 2001). Ésta es sin duda, una de las razones que hace necesaria la investigación sobre los patrones educativos y la 
aportación de datos actualizados sobre sus diferencias en relación con la edad y el sexo de los hijos.

El presente trabajo tiene por objetivo aportar nuevos datos sobre la percepción de la crianza que los hijos tienen sobre sus madres y padres. Se incluye una amplia muestra de sujetos con un extenso rango de edad, no siempre estudiado, incorporando el análisis tanto de hábitos de crianza positivos (e.g., comunicación, afecto) como negativos (control autoritario, hostilidad). Todo ello permitirá conocer de forma actualizada cuál es la percepción de nuestros menores sobre su crianza y, de acuerdo con la perspectiva de los hijos, cuál es el grado de tipificación por sexos y el ajuste evolutivo de dicha crianza a los diferentes grupos de edad.

Método

\section{Participantes}

La extracción de la muestra ha sido aleatoria por el método de conglomerados. Un total de 1182 sujetos participaron en el presente estudio, de los cuales el 45,3\% fueron varones y $54,7 \%$ mujeres. Sus edades estaban comprendidas entre los 8 y los 16 años $(\mathrm{M}=12,17$; DT $=1,61)$. Las diferentes edades se agruparon en tres rangos de edad desde 8 a 11 años $(n=427$; $36,2 \%)$; desde 12 a 13 años $(n=535 ; 45,2 \%)$, y desde 14 a 16 años $(n=220 ; 18,6 \%)$

La mayoría de los sujetos proceden de niveles socio-económicos medio $(43 \%)$ y medio-alto $(42,5 \%)$, en su mayoría primogénitos $(50,7 \%)$ o nacidos en segundo lugar $(34,4 \%)$ y con dos hermanos de media. El 87,8\% de los sujetos provienen de hogares en los que conviven ambos padres.

\section{Procedimiento}

Los sujetos fueron seleccionados aleatoriamente del conjunto de colegios de la ciudad de Madrid y su área metropolitana. Una vez seleccionados, se solicitó la autorización de los centros escolares y de los correspondientes padres. Se excluyeron los sujetos no autorizados y, a posteriori, aquéllos cuya madurez lectora era insuficiente. Las pruebas se administraban colectivamente en el grupo clase por psicólogos entrenados $\mathrm{y}$ en presencia del profesor. Las presentes medidas formaban parte de una recogida de datos más extensa, inserta en una amplia investigación sobre emociones infantiles.

\section{Instrumentos}

Cuestionario de comportamiento parental para niños (CRPBI; Child's Report of Parental Behavior Inventory) (Schaefer, 1965; adaptado al castellano por Carrasco, Holgado y del Barrio, 2007; Samper et al., 2006).
Este instrumento consta de 52 ítems, cada uno de los cuales se refiere a diferentes hábitos de crianza del padre y de la madre. Todos los ítems fueron evaluados mediante una escala tipo likert con tres niveles de respuesta: 1 (siempre), 2 (algunas veces); y 3 (nunca). El autoinforme valora por separado, pero con los mismos elementos, al padre y a la madre. Los factores analizados fueron los obtenidos por Carrasco et al. (2007) mediante análisis factorial exploratorio y confirmatorio. En total, resultaron tres factores de segundo orden (comunicación/ afecto, control y hostilidad/permisividad) y 6 de primer orden (afecto, comunicación, supervisión, control autoritario, hostilidad y permisividad/negligencia).

El factor de segundo orden, Comunicación-afecto, mide comportamientos parentales de implicación, cariño y atención (23 ítems; ej., "siempre escucha mis ideas y opiniones"; $\alpha=$ 0,86 para las madres y $\alpha=0,87$ para los padres). Este factor está compuesto por otros dos factores de primer orden: Afecto (11 ítems; ej., "a menudo me alaba"; $\alpha=0,81$ para las madres y $\alpha=0,77$ para los padres) y Comunicación (12 ítems; ej., "me da compresión cuando lo necesito"; $\alpha=0,79$ para las madres y $\alpha=0,79$ para los padres). El factor Control, de segundo orden, evalúa el grado y forma de controlar que los padres ejercen sobre las conductas de los hijos (13 ítems; ej., "quiere controlar todo lo que hago"; $\alpha=0,75$ para las madres y $\alpha=0,76$ para los padres). A su vez, este factor está formado por dos factores de primer orden, que especifican dos tipos de control. En primer lugar, el factor Control-Supervisión ( 8 ítems; ej., "siempre me está recordando las cosas que no me permite hacer"; $\alpha=0,70$ para las madres y $\alpha=0,76$ para los padres) y, en segundo lugar, el factor Control-Autoritario ( 8 ítems; ej., "casi siempre me castiga de alguna forma cuando soy malo/a"; $\alpha=0,66$ para las madres y $\alpha=0,57$ para los padres). Hostilidad/Negligencia es el último de los factores de segundo orden y evalúa las conductas parentales de hostilidad, rechazo, negligencia y permisividad (12 ítems; ej., "actúa como si yo fuera un estorbo"; $\alpha=0,70$ para las madres y $\alpha=0,68$ para los padres). Se compone de dos factores de primer orden: el factor Hostilidad, que evalúa el grado de rechazo, la falta de interés y respuesta hacia el hijo (6 ítems; ej., "a menudo dice que soy estúpido/a"; $\alpha=0,74$ para las madres y $\alpha=0,75$ para los padres), y el factor Permisividad/ Negligencia" que valora más específicamente la desatención y desinterés de los padres hacia los hijos (4 ítems; ej., "olvida darme las cosas que necesito"; $\alpha=0,65$ para las madres y $\alpha=$ 0,62 para los padres).

Resultados

Diferencias percibidas por los hijos sobre los hábitos de crianza maternos y paternos

Para analizar las diferencias entre los hábitos de crianza maternos y paternos percibidos por los hijos, se realizaron com- 
paraciones de medias entre padres y madres en cada uno de los factores de crianza. Las diferencias entre los dos grupos se calcularon a través de la t de Student. Como puede verse en la Tabla 1, las puntuaciones medias en la totalidad de los factores maternos fueron significativamente superiores a los paternos, con la excepción del factor Hostilidad, cuyas puntuaciones resultaron equiparables para madres y padres.

Tabla 1. Comparación de medias mediante T de Student entre los hábitos de crianza percibidos maternos y paternos.

\begin{tabular}{|c|c|c|c|c|}
\hline & & Media & D. T. & $t(1223)$ \\
\hline \multirow{2}{*}{ Comunicación/Afecto } & Madre & 56.62 & 6.44 & \multirow{2}{*}{$8.76^{* *}$} \\
\hline & Padre & 55.14 & 6.75 & \\
\hline \multirow{2}{*}{ Afecto } & Madre & 26.79 & 3.45 & \multirow{2}{*}{$8.29 * *$} \\
\hline & Padre & 26.03 & 3.52 & \\
\hline \multirow{2}{*}{ Comunicación } & Madre & 29.83 & 3.54 & \multirow{2}{*}{$7.69^{* *}$} \\
\hline & Padre & 29.11 & 3.77 & \\
\hline \multirow{2}{*}{ Control } & Madre & 25.06 & 4.36 & \multirow{2}{*}{$5.63^{* *}$} \\
\hline & Padre & 24.55 & 4.33 & \\
\hline \multirow{2}{*}{ Supervisión } & Madre & 14.98 & 3.01 & \multirow{2}{*}{$6.39 * *$} \\
\hline & Padre & 14.58 & 3.02 & \\
\hline \multirow{2}{*}{ Control autoritario } & Madre & 15.42 & 2.78 & \multirow{2}{*}{$2.63^{* *}$} \\
\hline & Padre & 15.26 & 2.79 & \\
\hline \multirow{2}{*}{ Hostilidad/Permisividad } & Madre & 18.22 & 3.58 & \multirow{2}{*}{$2.92^{* *}$} \\
\hline & Padre & 18.01 & 3.41 & \\
\hline \multirow{2}{*}{ Hostilidad } & Madre & 8.14 & 2.30 & \multirow{2}{*}{1.48} \\
\hline & Padre & 8.06 & 2.24 & \\
\hline \multirow{2}{*}{ Permisividad/Negligencia } & Madre & 6.66 & 2.05 & \multirow{2}{*}{$2.18^{* * *}$} \\
\hline & Padre & 6.57 & 1.92 & \\
\hline
\end{tabular}

$* * \mathrm{p}<0.001$

\section{Diferencias por sexo y edad: efectos principales}

El estudio de las diferencias por sexo y edad se llevó a cabo mediante dos análisis multivariados de la varianza (MANOVA), uno para las variables relativas a los hábitos de crianza paternos y otro a los maternos. El Modelo Líneal General correspondiente a los hábitos de crianza maternos resultó significativo para el sexo $[F(8,1093)=7,20, p=0,001)$ y la edad $[F$ $(16,2188)=10,35, p=0,001]$, pero no para la interacción $[F$ $(16,2188)=1,27, p=0,203]$. De igual forma ocurrió con el Modelo Lineal General correspondiente a los hábitos de crianza paternos, resultó significativo para el sexo $[F(8,1093)=9,21$, $p=0,001]$ y la edad $[F(16,2188)=8,51, p=0,001]$, pero no para la interacción $[F(16,2188)=1,09, p=0,351]$. A continuación se detallan los resultados correspondientes a los efectos principales de cada una de estas variables junto con los efectos interactivos de las mismas.

\section{Diferencias por sexo: efectos principales}

La Tabla 2 recoge las puntuaciones medias y las desviaciones típicas obtenidas en cada uno de los factores de crianza maternos y paternos según el sexo de los hijos. Como puede observarse, las niñas, frente a los niños, otorgan puntuaciones medias superiores tanto a sus madres como a sus padres en los factores de afecto y comunicación. En cambio, los niños puntúan más en los hábitos de crianza relacionados con la hostilidad y el control, tanto en relación con la supervisión como con el control autoritario. Por tanto, las chicas perciben, en mayor medida, a sus padres y madres más afectuosos, cercanos e implicados, mientras que los chicos los perciben más controladores, autoritarios permisivos y hostiles. Tanto en padres como en madres, el patrón de resultados se mostró bastante similar. Las diferencias en el factor global de comunicación se diluyen cuando se analizan los dos factores de primer orden incluidos en el mismo.

Tabla 2. Diferencias entre los hábitos de crianza percibidos sobre el padre y la madre en función del sexo de los hijos.

\begin{tabular}{|c|c|c|c|c|c|}
\hline \multirow[b]{2}{*}{$\begin{array}{l}\text { Hábitos de } \\
\text { crianza }\end{array}$} & & \multicolumn{2}{|c|}{ Madre } & \multicolumn{2}{|c|}{ Padre } \\
\hline & & $M(D T)$ & $F(1,1180)$ & $M(D T)$ & $F(1,1180)$ \\
\hline \multirow{2}{*}{$\begin{array}{l}\text { Comunicación/ } \\
\text { afecto }\end{array}$} & Varones & $55.67(6.59)$ & \multirow{2}{*}{ 23.04** } & $54.72(6.64)$ & \multirow{2}{*}{$4.69 *$} \\
\hline & Mujeres & $57.52(6.45)$ & & $55.60(7.06)$ & \\
\hline \multirow{2}{*}{ Afecto } & Varones & $26,16(3.47)$ & \multirow{2}{*}{$31.73 * *$} & $25.77(3.40)$ & \multirow{2}{*}{$5.98 *$} \\
\hline & Mujeres & $27,32(3.48)$ & & $26.29(3.73)$ & \\
\hline \multirow{2}{*}{ Comunicación } & Varones & $29.50(3.66)$ & \multirow{2}{*}{$10.51 * *$} & $28.95(3.84)$ & \multirow{2}{*}{2.53} \\
\hline & Mujeres & $30.19(3.54)$ & & $29.31(3.84)$ & \\
\hline \multirow{2}{*}{ Control } & Varones & $25.87(4.43)$ & \multirow{2}{*}{$36.27 * *$} & $25.47(4.37)$ & \multirow{2}{*}{48.56 ** } \\
\hline & Mujeres & $24.31(4.33)$ & & $23.70(4.25)$ & \\
\hline \multirow{2}{*}{ Supervisión } & Varones & $15.50(3.07)$ & \multirow{2}{*}{$32.28 * *$} & $15.17(3.05)$ & \multirow{2}{*}{$43.66 * *$} \\
\hline & Mujeres & $14.49(2.96)$ & & $14(2.96)$ & \\
\hline \multirow{2}{*}{$\begin{array}{l}\text { Control } \\
\text { autoritario }\end{array}$} & Varones & $15.92(2.81)$ & \multirow{2}{*}{$31.57 * *$} & $15.80(2.82)$ & \multirow{2}{*}{$36.14 * *$} \\
\hline & Mujeres & $15.00(2.77)$ & & $14.82(2.75)$ & \\
\hline \multirow{2}{*}{$\begin{array}{l}\text { Hostilidad/ } \\
\text { Negligencia }\end{array}$} & Varones & $18.79(3.85)$ & \multirow{2}{*}{$22.66^{* *}$} & $18.71(3.87)$ & \multirow{2}{*}{$40.61 * *$} \\
\hline & Mujeres & $17.78(3.37)$ & & $17.43(2.91)$ & \\
\hline \multirow{2}{*}{ Hostilidad } & Varones & $8.55(2.51)$ & \multirow{2}{*}{28.14 ** } & $8.54(2.58)$ & \multirow{2}{*}{$43.85 * *$} \\
\hline & Mujeres & $7.83(2.09)$ & & $7.67(1.85)$ & \\
\hline \multirow{2}{*}{$\begin{array}{l}\text { Permisividad/ } \\
\text { Negligencia }\end{array}$} & Varones & $6.83(2.04)$ & \multirow{2}{*}{ 7.34* } & $6.76(2.01)$ & \multirow{2}{*}{$10.79 * *$} \\
\hline & Mujeres & $6.50(2.10)$ & & $6.38(1.87)$ & \\
\hline
\end{tabular}

\section{Diferencias por edad: efectos principales}

Los descriptivos y significación de las diferencias en las dimensiones de crianza estudiadas en función de la edad de los hijos se recogen en la Tabla 3. Se establecieron tres grupos de edad, de acuerdo con la proximidad en sus niveles de desarrollo evolutivo: preadolescencia de 8 a 11 años; adolescencia media de 12 a 13 años; y adolescencia de 14 a 17 años.

Como puede apreciarse en la Tabla 3, la mayoría de las dimensiones de crianza son diferencialmente percibidas en función de la edad de los hijos. En concreto, los resultados indican que los hijos más pequeños muestran niveles medios superiores en los factores de afecto y comunicación, así como en el de control, particularmente en el de supervisión. Es decir, los hijos de menor edad perciben tanto a sus madres como a sus padres más cariñosos, comunicativos e implicados en la supervisión. 
Por el contrario, sólo los hábitos de crianza relacionados con la Hostilidad/Negligencia parental resultaron significativamente superiores en los grupos de más edad.

Tabla 3. Diferencias entre los hábitos de crianza percibidos sobre el padre y la madre en función de la edad de los hijos.

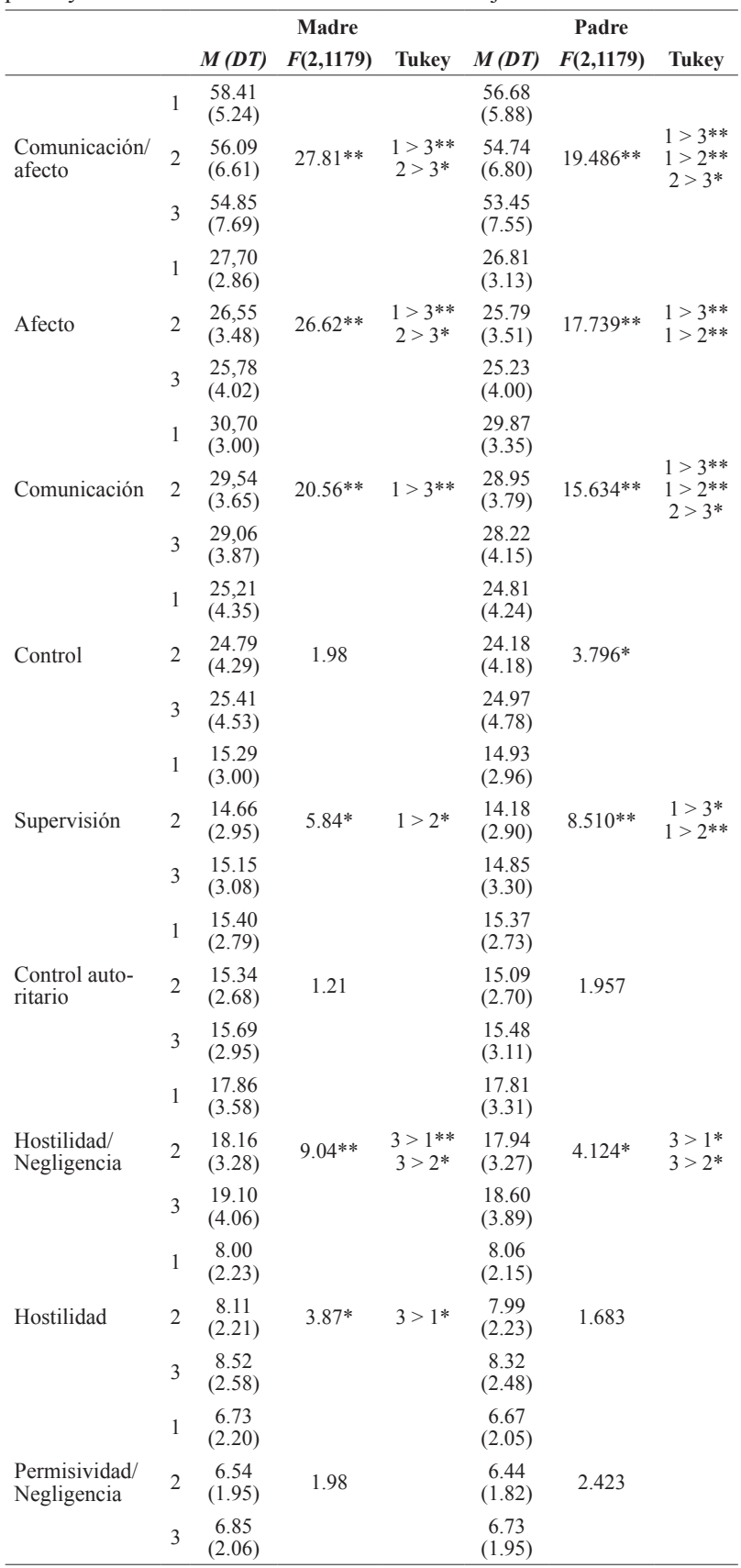

Nota: $1=8-11$ años; $2=12-13$ años; $3=14-17$ años.

$* \mathrm{p}<0.05 ; * \mathrm{p}<0.01$

Con el fin de obtener un análisis más pormenorizado de las diferencias grupales, se realizaron contrastes a posteriori mediante la prueba estadística de Tukey. Tal y como se recoge en la Tabla 3, en los factores relativos a la comunicación y el afecto así como al control, son los sujetos más pequeños los que establecen las diferencias, especialmente en los padres. Sin embargo, en los factores relativos a hostilidad y negligencia son los sujetos de mayor edad los responsables de las diferencias halladas.

Atendiendo al comportamiento diferencial entre padres y madres, como ocurría en los análisis por sexo, es destacable la similitud percibida por los hijos en ambos progenitores en función de la edad. No obstante, cuando consideramos la edad la tendencia anteriormente señalada es más patente entre los padres, es decir, parece que la edad establece mayor discriminación en la percepción de los hábitos de crianza paternos que maternos. Destacar tan sólo dos diferencias entre madre y padre: el control globalmente considerado en el padre y la hostilidad en la madre. En el primero, las diferencias se diluyen cuando se analizan las dimensiones de supervisión y control autoritario mostrando un patrón similar en padres y madres; y en el segundo, son los hijos de mayor edad los que perciben más hostilidad en la madre pero no en el padre.

Figura 1. Interacción sexo por edad en Comunicación/afecto materno.

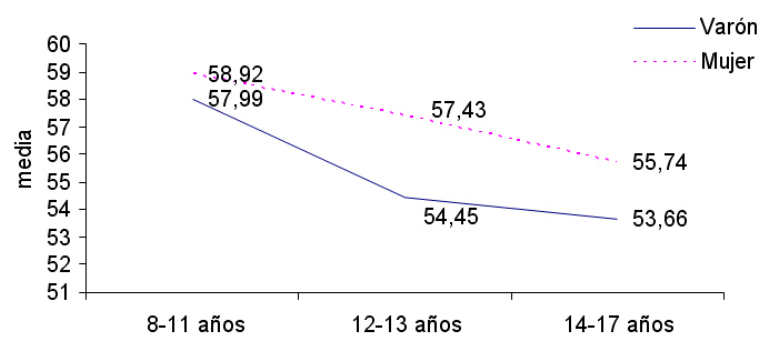

Figura 2. Interacción sexo por edad en Comunicación materno.

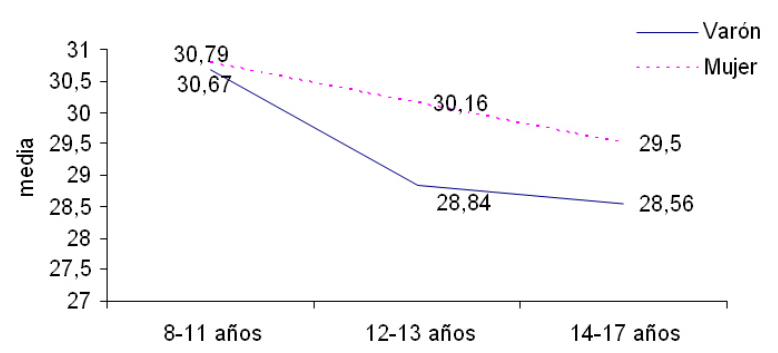

En relación con los hábitos de crianza paternos, la interacción resultó igualmente significativa para los factores comunicación/afecto $[F(2,1176)=3,280 ; p<0,05]$ y afecto $[F(2,1176)=$ $3.520 ; p<0,05)]$. En ambos casos, los varones tienden a percibir mayores niveles de comunicación y afecto paternos con la edad, en cambio, en las mujeres esta tendencia se invierte, a mayor edad la percepción de afecto y comunicación paternas tiende a disminuir (ver gráficos 3 y 4). 
Figura 3. Efecto de la interacción sexo por edad en la comunicación/ afecto paterno.

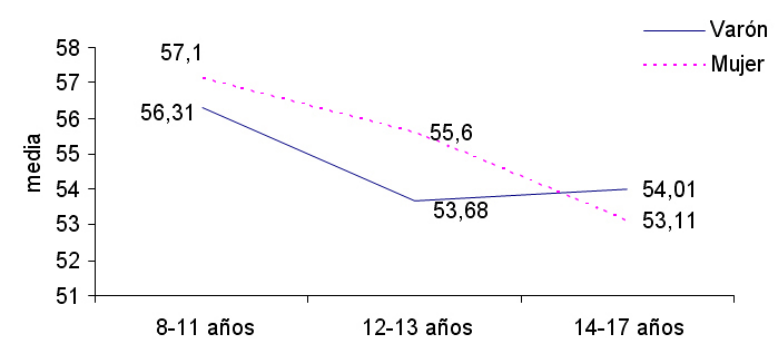

Figura 4. Efecto de la interacción sexo por edad en afecto paterno.

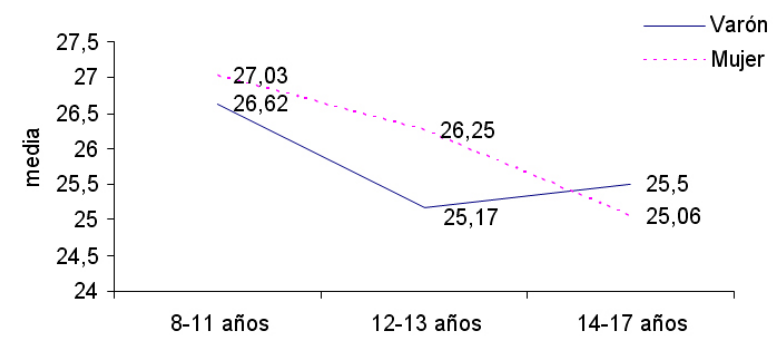

Discusión

El objetivo principal de esta investigación era aportar datos actuales sobre la percepción que los hijos tienen de las pautas de crianza paternas, según los sexos y el ajuste evolutivo de dichas pautas.

De acuerdo con la investigación previa, los presentes resultados constatan que la crianza paterna y materna se percibe diferencialmente por los hijos y que esta percepción parece estar claramente mediada por el sexo y la edad de éstos.

$\mathrm{Si}$ atendemos a las diferencias percibidas entre la madre y el padre, los hijos, en este caso independientemente del sexo y la edad, coinciden en valorar a sus madres con mayores niveles de comunicación, afecto, control y permisividad que a sus padres. Dato que viene a respaldar, aún en la actualidad, la mayor implicación y presencia de la mujer en la crianza de los hijos frente a los varones. Estas diferencias entre madres y padres, se han hallado por numerosos autores en población infantil y adolescente (Collin y Russell, 1991; Klein y O'Bryant, 1996; Paulson et al., 1991; Paulson y Sputa, 1996; Pipp et al., 1985; Rodrigo et al., 2005; Samper et al., 2006; Shek, 1998, 2000; Sorbring et al.; Youniss y Smollar, 1985). La mayor implicación y presencia de la mujer en la crianza explicaría tanto la elevada aparición de interacciones positivas entre hijos y madres (Fuentes, Motrico y Bersabé., 2001; Paulson y Sputa, 1996) como conflictivas (Collins y Russell, 1991; Laursen et al., 1998; Rodrigo et al., 2005; Russell y Russell, 1987), en comparación con las interacciones que muestran los padres con los hijos.
Un análisis más detallado de cada uno de los factores de crianza evaluados en este estudio, muestra, en consonancia con otros trabajos, que las madres se manifiestan más comunicativas, afectuosas, dialogantes, implicadas y preocupadas (Forehand y Nousiainen, 1993; Paulson et al., 1991; Paulson y Sputa, 1996; Samper et al., 2006; Youniss y Smollar, 1985) que los padres (Youniss y Smollar, 1985; Shek, 1995). A su vez, las madres emplean un mayor número de comportamientos de control, exigencias y estrategias de disciplina (Paulson y Sputa, 1996). En relación con los comportamientos hostiles y de rechazo, padres y madres son percibidos de forma similar, y esto también ha sido apoyado en trabajos previos (Chen, Huanfeng, Wang y Cen, 2001).

Cabría por tanto afirmar, que el estereotipo de "padre estricto y madre cariñosa" (Shek, 1998) sólo se sustenta parcialmente. Si bien las madres son más afectivas que los padres, éstos no son más exigentes o autoritarios que aquellas, tal y como informan otros autores (Youniss y Smollar, 1985; Collins y Russell, 1991; Klein y O'Bryant, 1996; Shek, 1995, 1998, 2000; Sorbring et al., 2003). Las madres, según la perspectiva de los hijos, además de mostrase más afectivas, cercanas e implicadas que los padres también se muestran más controladoras, autoritarias y permisivas.

Cuando el análisis de la crianza se hace en función del sexo y la edad de los hijos, las conclusiones adquieren numerosos matices. En el presente estudio, el sexo de los hijos media la percepción de la crianza materna y paterna. Las hijas son más sensibles al afecto, la cercanía e implicación, pero los hijos varones son más sensibles al control, la supervisión, la autoridad, la hostilidad y la permisividad, tanto de las madres como de los padres. Estos resultados son coincidentes con trabajos previos, los cuales muestran a las hijas más sensibles a la percepción de los hábitos de crianza maternos y paternos relacionados con la comunicación y el afecto (Fuentes et al., 2001; Gaylord et al., 2003; Mestre et al., 2007; Russell y Russell, 1987; Shek, 1995, 1998, 2000) y, por el contrario, a los hijos varones más sensibles a la percepción del control, la disciplina y la hostilidad (Collins y Russell, 1991; Fuentes et al., 2001; Mestre et al., 2007). No obstante, como ya apuntábamos, la literatura no está exenta de controversia en relación con estos resultados (Dornbusch, Ritter, Herbert, Roberts y Fraleigh, 1987; Forehand y Nousiaien, 1993; Musitu y Cava, 2001; Shek, 1998; Smetana, 1995); controversia que ha sido atribuida a los cambios que en los últimos años han podido acontecer en el estilo educativo de padres y madres (Lanz et al., 2001) hacia una educación más igualitaria.

No obstante, esta tendencia al equilibrio en la crianza de padres y madres en función del sexo de los hijos, no ha sido corroborada en los presentes resultados. Quizás esta última afirmación, de acuerdo con nuestros resultados, deba ser matizada con relación a la comunicación de los padres, la cual se ha mostrado equiparable tanto para los hijos (varones) como para las hijas. En lo que resta (variables de control y hostilidad), los 
resultados aquí mostrados avalan la creencia popular de que padres y madres siguen siendo aún más rígidos, controladores y críticos con los hijos varones que con las hijas, así como más hostiles y permisivos.

En relación con la edad, los resultados obtenidos muestran que los hijos más pequeños son los que perciben mayor cariño, implicación, comunicación y supervisión tanto de los padres como de las madres. Por el contrario, los hijos de más edad perciben en mayor medida comportamientos parentales de hostilidad y negligencia, especialmente en las madres. Como se ha recogido por otros autores, el apoyo y el control de los padres y madres disminuye a medida que aumenta la edad de los hijos (Feldman y Gehring; 1988; Furman y Buhrmester, 1992; Lanz et al., 2001; Litovsky y Dusek, 1985; McNally et al.,, 1991; Musitu y Cava, 2001; Paikoff y Brooks-Gunn, 1991; Paulson y Sputa, 1996; Shek, 2000; Spera, 2005; Steinberg y Silverger, 1987). También con el incremento de la edad, padres y madres muestran un mayor número de comportamientos de rechazo, negligencia, hostilidad y permisividad (Holmbeck y Hill, 1991; Laursen, Coy y Collins, 1998). Seguramente, estos cambios estén estrechamente relacionados con las necesidades y demandas asociadas a los cambios evolutivos de los hijos desde la infancia a la adolescencia y al reajuste que ello supone en la dinámica familiar.

Es destacable que el patrón mostrado por los padres y las madres en relación con el sexo y la edad, es bastante similar. Los comportamientos de los padres y las madres, siempre según la perspectiva de los hijos, es modulado en la misma dirección por la edad y el sexo. Sin embargo, respecto al sexo, la comunicación paterna es similar en hijos e hijas mientras que la comunicación materna es superior en las hijas. Respecto a la edad, la hostilidad paterna es similar en hijos e hijas de cualquier edad, mientras que la hostilidad materna es mayor entre los hijos e hijas de más edad.

El análisis de las interacciones sexo por edad, sólo resultó significativo en los hábitos de crianza relacionados con la comunicación y el afecto. Las hijas y los hijos cuando son pequeños perciben de forma similar tanto a los padres como a las madres, sin embargo, cuando crecen las chicas perciben más afecto que los chicos. Estos datos son coherentes con resultados anteriores en los que el mayor afecto es percibido por las hijas y, sobre todo, por las de menor edad. A medida que los hijos e hijas se acercan a la adolescencia, disminuye progresivamente la percepción de afecto parental. Especialmente, disminuye la comunicación/ afecto materna, para los chicos, y la paterna para las chicas.

Finalmente, hay que señalar algunas de las limitaciones de este trabajo. Una de ellas está relacionada con el uso de una única fuente para la evaluación de la crianza de los padres. Aunque, los hijos son informantes válidos y fiables, las conclusiones de estos resultados sólo pueden sostenerse desde la perspectiva de los hijos y de sus percepciones. La consideración de otras fuentes informantes como la de los padres o la procedente de fuentes externas permitirían el análisis de la crianza desde otras perspectivas. Por otra parte, el rango de edad de los sujetos estudiados no ha permitido el estudio de la percepción más allá de la adolescencia, dónde posiblemente la tendencia de los cambios percibidos sería más clara. Por último, destacar que aunque los datos proceden de una muestra amplia y representativa, ésta se circunscribe exclusivamente a la población madrileña que, aún siendo un punto de encuentro de individuos procedentes de diversas partes del país y de otras culturas, puede no ser totalmente equiparable al de otros ámbitos nacionales.

\section{Referencias}

Achenbach, T. M. y McDonough, S. H. (1987). Empirically based assessment of child and adolescent psychopathology. Practical applications. Newbury Park, Ca: Sage.

Carrasco, M. A., Holgado, P. y Del Barrio M. V. (2007). Análisis de la estructura del cuestionario de comportamiento parental para niños (CRPBI) en población española. Revista Iberoamericana de Diagnóstico y Evaluación Psicológica, 24, 95-120.

Chen, X., Wu, H., Chen, H., Van, L., y Cen G. (2001), Parenting practices and aggression behavior in Chinese children. Parenting: Science and Practice, 1, 159-184.

Cole, D. A., Martin, J. M., Powers, B. y Truglio, R. (1996). Modeling causal relations between academic and social competence and depression: a multitrait-multimethod longitudinal study of children. Journal of Abnormal Psychology, 105, 258-270.

Collins, W. y Russell, G. (1991). Mother-child and father-child relationships in middle childhood and adolescence: a developmental analysis. Developmental Review, 11, 99-136.

Del Barrio, M. V. (1990). Situación actual de la evaluación de la depresión infantil. Evaluación Psicológica/European Psychological Assessment, 6(2), 171-209.

Del Barrio, M. V. (2002). Método de la evaluación psicológica y su aplicación al niño y al adolescente. En V. del Barrio (Ed.), Evaluación psicológica en la infancia y la adolescencia. Madrid: UNED

Dornbusch, S. M., Ritter, P.L., Leiderman, P.H., Roberts, D.F. y Fraleigh, J. (1987). The relation of parenting style to adolescent School performance. Child Development, 58, 1244-1257.

Epkins, C. (1993). A preliminary comparison of teacher ratings and child self-report of depression, anxiety, and aggression in inpatient and elementary school samples. Journal of Abnormal Child Psychology, 21, 649-661.

Feldman, S. y Gehring, T. (1988). Changing perceptions of family cohesion and power across adolescence. Child Development, 59, 1034-1045.

Forehand, R. y Nousiainen, S. (1993). Maternal and paternal parenting: Critical dimensions in adolescent functioning. Journal of Family Psychology, 7, 213-221. 
Fuentes, M. J., Motrico, E., y Bersabé, R. M. (2001). Diferencias entre padres y adolescentes en la percepción del estilo educativo parental: afecto y normas-exigencias. Apuntes de Psicología, 19, 235-249.

Furman, W. y Buhrmester, D. (1992). Age and sex differences in perceptions of networks of personal relationships. Child Development, 63, 103-115.

Gaylord, N. K., Kitzmann, M. y Coleman, J. K. (2003). Parent and children's perceptions of parental behaviour: associations with children's psychological adjustment in the classroom. Parenting: Science and Practice. 3(1), 23-47.

Gonzales, N. Cauce, A., Mason y Craig A. (1996). Interobserver agreement in the assessment of parental behavior and parent-adolescent conflict: African American mothers, daughters, and independent observers. Child Development, 67(4), 1483-1498.

Grusec, J. y Goodnow, J. (1994). Impact of parental discipline methods on the child's internalization of values: A reconceptualization of current points of view. Developmental Psychology, 30(1), 4-19.

Hines, D., Kantor, G. y Holt, M. (2006) Similarities in siblings' experiences of neglectful parenting behaviours. Child Abuse and Neglect, 30, 619-637.

Holmbeck, GN., y Hill, J.P. (1991). Conflictive engagement, positive affect and menarche in families with seventh grade girls. Child Development, 62, 1030-1048.

Ivanova, M. e Israel, A. (2006). Family stability as a protective factor against psychopathology for urban children receiving psychological services. Journal of Clinical Child and Adolescent Psychology, 35, 564-570.

Jané, M., Araneda, N., Valero, S. y Doménech, E. (2000). Evaluación de la sintomatología depresiva del preescolar: correspondencia entre los informes de padres y de maestros. Psicothema, 12, 212-215.

Klein, H. y O'Bryant, K. (1996). Recalled parental authority style and self-perception in college men and woman. Journal of Genetic Psychology, 157, 5-17.

Klein, R. G. (1991). Parent-child agreement in clinical assessment of anxiety and other psychopathology: a review. Journal of Anxiety Disorders, 5, 187-198.

Lanz, M., Scabini, E., Vermulst, A. A. y Gerris, J. R. (2001). Congruence on child rearing in families with early adolescent and middle adolescent children. International Journal of Behavioral Development, 25, 133-139.

Laursen, B., Coy, K., y Collins, W. (1998). Reconsidering changes in parent-child conflict across adolescence: A metaanalysis. Child Development, 69, 817-832.

Laursen, B., Coy, K.C., y Collins, W. A. (1998). Reconsidering changes in parent-child conflict across adolescent: A metaanalysis. Child Development, 69, 817-832.

Litovsky, V. y Dusek, J. (1985). Perceptions of child rearing and self-concept development during the early adolescent years. Journal of Youth and Adolescence, 14, 373-387.
McNally, S., Eisenberg, N. y Harris, J. (1991) Consistency and change in maternal child-rearing practices and values: a longitudinal study. Child Development, 62, 191-198.

Mestre, M. V, Samper, P., Tur, A. y Díez, I. (2001). Estilos de crianza y desarrollo prosocial de los hijos. Revista de Psicología General y Aplicada, 54, 691-703.

Morris, A., Silk, J., Steinberg, L., Sessa, F., Avenevoli, S. y Essex, M. (2002). Temperamental vulnerability and negative parenting as interacting of child adjustment. Journal of Marriage and Family, 64, 461-471.

Musitu, G. y Cava, M. J. (2001). El rol del apoyo social en el ajuste de los adolescentes. Intervención Psicosocial, 12, 179-192.

Paikoff, R. y Brooks-Gunn, J. (1991). Do Parent-child relationships change during puberty? Psychological Bulletin, 110, 47-66.

Paulson, E. y Sputa, L. (1996) Patterns of parenting during adolescence: perception of adolescents and parents. Adolescence, 31, 369-381.

Paulson, E., Hill, J. y Holmbeck (1991). Distinguishing between perceived closeness and parental warmth in families with seventh-grade boys and girls. Journal of Early Adolescence, 11(2), 276-293.

Paulson, S. (1994). Relations of parenting style and parental involvement with ninth-grade students' achievement. Journal of Early Adolescence, 14, 2, 250-267.

Pipp, S., Shaver, P., Jennings, Lamborn, S. y Fishers, K. W. (1985). Adolescence's theories about the development of their relationships with parents. Journal of Personality and Social Psychology, 48, 991-1001.

Roa, L. y Del Barrio, V. (2001). Adaptación del cuestionario de crianza parental (PCRI-A Gerard, 1994) a la población española. Revista Latinoamericana de Psicología, 33, 329-341.

Roa, L. y Del Barrio, V. (2002). Cuestionario de percepción de crianza para niños y adolescentes. Psicología Educativa, 8 , 1,37-51.

Rodrigo, M., García, M., Márquez, M. y Triana, B. (2005). Discrepancias entre padres e hijos adolescentes en la frecuencia percibida e intensidad emocional en los conflictos familiares. Estudios de Psicología, 26, 1, 21-34.

Russell, G. y Russell, A. (1987). Mother-child and father-child relationships in middle childhood. Child Development, 58, 1573-1585.

Samper, P Cortés, M., Mestre, V., Nácher, M. y Tur, A. (2006). Adaptación del Child's Report of Parent Behavior Inventory (Schaefer, 1965) a población española. Psicothema, 18, 263-271

Schaefer, E. S. (1965). Children's reports of parental behaviour: an inventory. Child Development, 36(2), 413-424.

Shek, D. (1995). Adolescent perceptions of parental and maternal parenting styles in a Chinese context. Journal of Psychology: Interdisciplinary and Applied, 132, 527-537. 
Shek, D. (1998). Perceptions of parenting styles and parentadolescent conflict in adolescents with low academic achievement in Hong Kong. Social Behavior and Personality, 26, 89-98.

Shek, D. (2000). Differences between fathers and mothers in the treatment of, and relationship with, their teenage children: perceptions of Chinese adolescents. Adolescence, 35, 135-146.

Smetana. J. (1995). Parenting styles and conceptions of parental authority during adolescence. Child Development, 66, 299-316.

Sorbring, E., Rödholm-Funnemark, M. y Palmerus, K. (2003) Boys'and gilrs' perceptions of parental discipline in transgression situations. Infant and Child Development, 12, 53-69.

Spera, C. (2005). A review of the relationship among parenting practices, parenting styles, and adolescent school achievement. Educational Psychology Review, 17, 25-146.
Steinberg, L. (2001). We know some things: parent-adolescent relationships in retrospect and prospect. Journal of Research on Adolescence, 11, 1-19.

Steinberg, L. y Silverger, S.B. (1987). Influences on marital satisfaction during the middle stages of the family life cycle. Journal of Marriage and Family, 49, 751-760.

Youniss, J. y Smollar, J. (1985). Adolescent relations with mothers, fathers and friends. Chicago: University of Chicago Press.
Fecha de recepción: 5 de marzo de 2009

Fecha de aceptación 19 de marzo de 2009 